\title{
FABER-KRAHN INEQUALITY FOR ANISOTROPIC EIGENVALUE PROBLEMS WITH ROBIN BOUNDARY CONDITIONS
}

\author{
FRANCESCO DELLA PIETRA AND NUNZIA GAVITONE
}

\begin{abstract}
In this paper we study the main properties of the first eigenvalue $\lambda_{1}(\Omega)$ and its eigenfunctions of a class of highly nonlinear elliptic operators in a bounded Lipschitz domain $\Omega \subset \mathbb{R}^{n}$, assuming a Robin boundary condition. Moreover, we prove a Faber-Krahn inequality for $\lambda_{1}(\Omega)$.
\end{abstract}

\section{INTRODUCTION}

Let $\Omega$ be a bounded Lipschitz domain in $\mathbb{R}^{n}, n \geq 2$. This paper is devoted to the study of the following problem:

$$
\lambda_{1}(\Omega)=\min _{\substack{u \in W^{1, p}(\Omega) \\ u \neq 0}} J(u)
$$

where

$$
J(u)=\frac{\int_{\Omega}[H(D u)]^{p} d x+\beta \int_{\partial \Omega}|u|^{p} H(\nu) d \sigma}{\int_{\Omega}|u|^{p} d x},
$$

$1<p<+\infty, \nu$ is the outer normal to $\partial \Omega$, and $\beta$ is a fixed positive number. Moreover, we suppose that $H$ is a sufficiently smooth norm of $\mathbb{R}^{n}$ (see Sections 2 and 3 for the precise assumptions). The minimizers of (1.1) satisfy the equation

$$
-\operatorname{div}\left([H(D u)]^{p-1} H_{\xi}(D u)\right)=\lambda_{1}(\Omega)|u|^{p-2} u \quad \text { in } \Omega,
$$

with Robin conditions on the boundary:

$$
[H(D u)]^{p-1} H_{\xi}(D u) \cdot \nu+\beta H(\nu)|u|^{p-2} u=0 \quad \text { on } \partial \Omega .
$$

The operator in (1.3) reduces to the $p$-Laplacian when $H$ is the Euclidean norm of $\mathbb{R}^{n}$. For a general norm $H$, it is an anisotropic, highly nonlinear operator, and it has attracted an increasing interest in last years. We refer, for example, to [1, 20, 24] $(p=2)$ and [4, 6, 19, 22] $(1<p<+\infty)$ where Dirichlet boundary conditions are considered. Moreover, for Neumann boundary values see, for instance, [18,36] $(p=2)$, while overdetermined problems are studied in [12,35] $(p=2)$. In this paper we are interested in considering the eigenvalue problem (1.3) with the Robin boundary conditions (1.4). In particular, our main objective is to obtain a Faber-Krahn inequality by studying the shape optimization problem

$$
\min _{|\Omega|=m} \lambda_{1}(\Omega)
$$

among all the Lipschitz domains with given measure $m>0$. To study problem (1.5), we first have to investigate the basic properties of the first eigenvalue and of the relative eigenfunctions of (1.3),(1.4), as existence, sign, simplicity and regularity.

Date: August 2, 2018.

2010 Mathematics Subject Classification. 35P15,35P30,35J60 .

Key words and phrases. Eigenvalue problems, nonlinear elliptic equations, Faber-Krahn inequality, Wulff shape. 
In the Euclidean case, problem (1.1) reduces to

$$
\lambda_{1, \mathcal{E}}(\Omega)=\min _{\substack{u \in W^{1, p}(\Omega) \\ u \neq 0}} \frac{\int_{\Omega}|D u|^{p} d x+\beta \int_{\partial \Omega}|u|^{p} d \sigma}{\int_{\Omega}|u|^{p} d x},
$$

and the minimizers satisfy the problem

$$
\begin{cases}-\operatorname{div}\left(|D u|^{p-2} D u\right)=\lambda_{1, \mathcal{E}}(\Omega)|u|^{p-2} u & \text { in } \Omega, \\ |D u|^{p-2} \frac{\partial u}{\partial \nu}+\beta|u|^{p-2} u=0 & \text { on } \partial \Omega .\end{cases}
$$

In such a case, problem (1.5) has been first investigated by Bossel for $p=2$, when $\Omega$ varies among smooth domains of $\mathbb{R}^{2}$ with fixed measure. More precisely, in [7] she proved that

$$
\lambda_{1, \mathcal{E}}(\Omega) \geq \lambda_{1, \mathcal{E}}(B),
$$

where $B$ is a disk such that $|B|=|\Omega|$. This result has been generalized to any dimension $n \geq 2$ for Lipschitz domains in [17. As regards the case $1<p<+\infty$, the inequality (1.6) has been proved by [16 for smooth domains, and by 9 in the case of Lipschitz domains. The equality cases are also addressed in 9, 16. As regards the case $\beta<0$, we refer the reader to [25] and the references therein.

In the anisotropic case, our result reads as follows. Let $H^{o}$ be the polar function of $H$, and denote by $\mathcal{W}_{R}$ the Wulff shape, that is the $R$-sublevel set of $H^{o}$, such that $\left|\mathcal{W}_{R}\right|=|\Omega|$ (see Section 2 for the definitions). If $\Omega \neq \mathcal{W}_{R}$ is a Lipschitz set of $\mathbb{R}^{n}$, then

$$
\lambda_{1}(\Omega)>\lambda_{1}\left(\mathcal{W}_{R}\right) .
$$

Hence, the unique minimizer of (1.5) is the Wulff shape. Such result relies in the so-called anisotropic isoperimetric inequality (see for example [1]), and it is in agreement with the Faber-Krahn inequality for the first eigenvalue of (1.3) in the homogeneous Dirichlet case (see [4]).

As a matter of fact, we may ask if the first eigenvalue $\lambda_{1}(\Omega)$ is bounded from above in terms of the Lebesgue measure of $\Omega$. Indeed, in the Euclidean setting, this is the case for the first nonvanishing Neumann Laplacian eigenvalue (see [37], and also [8, 11] for related results), but this does not happen for the first Dirichlet Laplacian eigenvalue. In this order of ideas, by a result given in [29] it follows that the first Robin Laplacian eigenvalue among the sets of fixed measure is unbounded from above. Here we prove a lower bound for the first eigenvalue $\lambda_{1}(\Omega)$ of our anisotropic Robin problem in a convex set $\lambda_{1}(\Omega)$ in terms of the anisotropic inradius of $\Omega$. This will imply that, among all Lipschitz sets with fixed measure $m>0$,

$$
\sup _{|\Omega|=m} \lambda_{1}(\Omega)=+\infty .
$$

The paper is organized as follows. In Section 2, we recall some basic definitions and properties of $H$ and of its polar function $H^{o}$. In Section 3, we state and prove some properties of the first eigenvalue of (1.3), (1.4). More precisely, under suitable assumptions on $H$, we show that there exists a first eigenvalue $\lambda_{1}(\Omega)$ which is simple. Moreover, we prove that the first eigenfunctions are in $C^{1, \alpha}(\Omega) \cap C(\bar{\Omega})$, for some $0<\alpha<1$. Furthermore, a solution of the eigenvalue problem is a first eigenfunction if and only if it has a fixed sign. In Section 4 we investigate the eigenvalue problem when $\Omega$ is a Wulff shape, while in Section 5 we give a representation formula for $\lambda_{1}(\Omega)$ by means of the level sets of the first eigenfunctions. Using such results, in Section 6 we state precisely the main result and give a proof. 


\section{Notation AND PRELiminaRies}

Let $H: \mathbb{R}^{n} \rightarrow\left[0,+\infty\left[, n \geq 2\right.\right.$, be a $C^{2}\left(\mathbb{R}^{n} \backslash\{0\}\right)$ function such that

$$
H(t \xi)=|t| H(\xi), \quad \forall \xi \in \mathbb{R}^{n}, \forall t \in \mathbb{R},
$$

and such that any level set $\left\{\xi \in \mathbb{R}^{n}: H(\xi) \leq t\right\}$, with $t>0$ is strictly convex.

Moreover, suppose that there exist two positive constants $a \leq b$ such that

$$
a|\xi| \leq H(\xi) \leq b|\xi|, \quad \forall \xi \in \mathbb{R}^{n} .
$$

Remark 2.1. We stress that the homogeneity of $H$ and the convexity of its level sets imply the convexity of $H$. Indeed, by (2.1), it is sufficient to show that, for any $\xi_{1}, \xi_{2} \in \mathbb{R}^{n} \backslash\{0\}$,

$$
H\left(\xi_{1}+\xi_{2}\right) \leq H\left(\xi_{1}\right)+H\left(\xi_{2}\right) .
$$

By the convexity of the level sets, we have

$$
\begin{aligned}
& H\left(\frac{\xi_{1}}{H\left(\xi_{1}\right)+H\left(\xi_{2}\right)}+\frac{\xi_{2}}{H\left(\xi_{1}\right)+H\left(\xi_{2}\right)}\right)= \\
& \quad=H\left(\frac{H\left(\xi_{1}\right)}{H\left(\xi_{1}\right)+H\left(\xi_{2}\right)} \frac{\xi_{1}}{H\left(\xi_{1}\right)}+\frac{H\left(\xi_{2}\right)}{H\left(\xi_{1}\right)+H\left(\xi_{2}\right)} \frac{\xi_{2}}{H\left(\xi_{2}\right)}\right) \leq 1,
\end{aligned}
$$

and by (2.1) we get (2.3).

We define the polar function $H^{o}: \mathbb{R}^{n} \rightarrow[0,+\infty[$ of $H$ as

$$
H^{o}(v)=\sup _{\xi \neq 0} \frac{\xi \cdot v}{H(\xi)} \text {. }
$$

It is easy to verify that also $H^{o}$ is a convex function which satisfies properties (2.1) and (2.2). Furthermore,

$$
H(v)=\sup _{\xi \neq 0} \frac{\xi \cdot v}{H^{o}(\xi)}
$$

The set

$$
\mathcal{W}=\left\{\xi \in \mathbb{R}^{n}: H^{o}(\xi)<1\right\}
$$

is the so-called Wulff shape centered at the origin. We put $\kappa_{n}=|\mathcal{W}|$, where $|\mathcal{W}|$ denotes the Lebesgue measure of $\mathcal{W}$. More generally, we denote with $\mathcal{W}_{r}\left(x_{0}\right)$ the set $r \mathcal{W}+x_{0}$, that is the Wulff shape centered at $x_{0}$ with measure $\kappa_{n} r^{n}$, and $\mathcal{W}_{r}(0)=\mathcal{W}_{r}$.

The following properties of $H$ and $H^{o}$ hold true (see for example [3]):

$$
\begin{gathered}
H_{\xi}(\xi) \cdot \xi=H(\xi), \quad H_{\xi}^{o}(\xi) \cdot \xi=H^{o}(\xi), \\
H\left(H_{\xi}^{o}(\xi)\right)=H^{o}\left(H_{\xi}(\xi)\right)=1, \quad \forall \xi \in \mathbb{R}^{n} \backslash\{0\}, \\
H^{o}(\xi) H_{\xi}\left(H_{\xi}^{o}(\xi)\right)=H(\xi) H_{\xi}^{o}\left(H_{\xi}(\xi)\right)=\xi, \quad \forall \xi \in \mathbb{R}^{n} \backslash\{0\} .
\end{gathered}
$$

Definition 2.1 (Anisotropic area functional and perimeter ( [2,10])). Let $M$ be an oriented $(n-1)$-dimensional hypersurface in $\mathbb{R}^{n}$. The anisotropic area functional of $M$ is

$$
\sigma_{H}(M):=\int_{M} H(\nu) d \sigma
$$

where $\nu$ denotes the outer normal to $M$ and $\sigma$ is the $(n-1)$-dimensional Hausdorff measure.

The anisotropic area of a set $M$ is finite if and only if the usual Euclidean hypersurface area $\sigma(M)$ is finite. Indeed, by property (2.2) we have that

$$
\alpha \sigma(M) \leq \sigma_{H}(M) \leq \gamma \sigma(M)
$$


An isoperimetric inequality for the anisotropic area holds, namely for $K \subset M$ open set of $\mathbb{R}^{n}$ with Lipschitz boundary,

$$
\sigma_{H}(\partial K) \geq n \kappa_{n}^{\frac{1}{n}}|K|^{1-\frac{1}{n}}
$$

and the equality holds if and only if $K$ is homothetic to a Wulff shape (see for example [10, [15], 26], [1]). We stress that in [21] an isoperimetric inequality for the anisotropic relative perimeter in the plane is studied.

Let $\Omega$ be a bounded open set of $\mathbb{R}^{n}$, and $d_{H}(x)$ the anisotropic distance of a point $x \in \Omega$ to the boundary $\partial \Omega$, that is

$$
d_{H}(x)=\inf _{y \in \partial \Omega} H^{o}(x-y) .
$$

By the property (2.6), the distance function $d_{H}(x)$ satisfies

$$
H\left(\operatorname{Dd}_{H}(x)\right)=1 .
$$

Finally, we recall that when $\Omega$ is convex $d_{H}(x)$ is concave. In a natural way, the anisotropic inradius of a convex, bounded open set $\Omega$ is the value

$$
R_{H, \Omega}=\sup \left\{d_{H}(x), x \in \Omega\right\}
$$

For further properties of the anisotropic distance function we refer the reader to [13].

\section{The FIRSt EIGENVALUe PROBlem}

In this section we prove some properties of the minimizers of (1.1), which are the weak solutions of the following Robin boundary value problem:

$$
\begin{cases}-\operatorname{div}\left(F_{p}(D u)\right)=\lambda_{1}(\Omega)|u|^{p-2} u & \text { in } \Omega \\ F_{p}(D u) \cdot \nu+\beta H(\nu)|u|^{p-2} u=0 & \text { on } \partial \Omega .\end{cases}
$$

where

$$
F_{p}(D u):=[H(D u)]^{p-1} H_{\xi}(D u) .
$$

For weak solution of problem (3.1) we mean a function $u \in W^{1, p}(\Omega)$ such that

$$
\int_{\Omega} F_{p}(D u) \cdot D \psi d x+\beta \int_{\partial \Omega} u^{p-1} \psi H(\nu) d \sigma=\lambda_{1}(\Omega) \int_{\Omega}|u|^{p-2} u \psi d x, \quad \psi \in W^{1, p}(\Omega) .
$$

Obviously, $\lambda_{1}(\Omega)$ in (1.1) (and then in (3.1)) depends also on $\beta$. In general, we will consider $\beta>0$ fixed. Anyway, when it will be necessary, to emphasize the dependence on $\beta$ we will denote the first eigenvalue of (3.1) with $\lambda_{1}(\Omega, \beta)$.

For the Euclidean case we refer to [31], where the eigenvalue problem for the $p$-Laplacian under several boundary conditions is considered.

From now on, we assume that $H$ is a convex function as in Section 2, assuming also that it verifies the following hypothesis:

$$
H \in C^{2}\left(\mathbb{R}^{n} \backslash\{0\}\right) \text {, with } \sum_{i, j=1}^{n} \frac{\partial}{\partial \xi_{j}}\left([H(\eta)]^{p-1} H_{\xi_{i}}(\eta)\right) \xi_{i} \xi_{j} \geq \gamma|\eta|^{p-2}|\xi|^{2},
$$

for some positive constant $\gamma$, for any $\eta \in \mathbb{R}^{n} \backslash\{0\}$ and for any $\xi \in \mathbb{R}^{n}$.

Theorem 3.1. There exists a function $u_{p} \in C^{1, \alpha}(\Omega) \cap C(\bar{\Omega})$ which realizes the minimum in (1.1), and satisfies the problem (3.1). Moreover, $\lambda_{1}(\Omega)$ is the first eigenvalue of (3.1), and the first eigenfuctions are positive (or negative) in $\Omega$. 
Proof. The proof makes use of standard arguments. We briefly recall the main steps. The direct method of the Calculus of Variations guarantees that the infimum in (1.1) is attained at a function $u_{p} \in W^{1, p}(\Omega)$. We may assume that $u_{p} \geq 0$, being also $\left|u_{p}\right|$ a minimizer in (1.1). Moreover, the function $u_{p}$ is a weak solution of (3.1). In order to obtain that $u_{p} \in C^{1, \alpha}(\Omega) \cap C(\bar{\Omega})$, we first claim that a $L^{\infty}$-estimate for $u_{p}$ holds. To get the claim, we take $\varphi=\left[T_{M}\left(u_{p}\right)\right]^{k p+1}$ as test function, with $k, M$ positive numbers, and $T_{M}(s)=\min \{s, M\}$, $s \geq 0$. Using (2.5) and (2.2), we easily get

$$
\begin{aligned}
& \alpha(k p+1) \int_{u_{p} \leq M}\left|D u_{p}\right|^{p} u_{p}^{k p} d x \leq \\
& \leq \int_{\Omega} F_{p}\left(D u_{p}\right) \cdot D \varphi d x+\beta \int_{\partial \Omega} u_{p}^{p-1} \varphi H(\nu) d \sigma \leq \\
& \quad \leq \lambda_{1}(\Omega) \int_{\Omega} u_{p}^{p(k+1)} d x,
\end{aligned}
$$

and then

$$
\int_{\Omega}\left|D T_{M}\left(u_{p}\right)^{k+1}\right|^{p} d x+\int_{\Omega}\left[T_{M}\left(u_{p}\right)\right]^{p(k+1)} d x \leq\left(\frac{(k+1)^{p}}{\alpha(k p+1)} \lambda_{1}(\Omega)+1\right) \int_{\Omega} u_{p}^{p(k+1)} d x .
$$

Applying the Sobolev inequality and the Fatou lemma, we get that

$$
\left\|u_{p}\right\|_{(k+1) p^{*}} \leq S^{\frac{1}{k+1}}\left(\frac{(k+1)^{p}}{k p+1} \frac{\lambda_{1}(\Omega)}{\alpha}+1\right)^{\frac{1}{p(k+1)}}\left\|u_{p}\right\|_{(k+1) p},
$$

where $S$ is the Sobolev constant. Using the standard Moser iteration technique for the $L^{p_{-}}$ norms, we get the claim. For sake of completeness, we give the complete proof (see also [27]).

First of all, we have that there exists a constant $c$ independent of $k$ such that

$$
\left(\frac{(k+1)^{p}}{k p+1} \frac{\lambda_{1}(\Omega)}{\alpha}+1\right)^{\frac{1}{p \sqrt{k+1}}} \leq c .
$$

Then,

$$
\left\|u_{p}\right\|_{(k+1) p^{*}} \leq S^{\frac{1}{k+1}} c^{\frac{1}{\sqrt{k+1}}}\left\|u_{p}\right\|_{(k+1) p} .
$$

Choosing $k_{n}$ in (3.4) such that $\left(k_{1}+1\right) p=p^{*}$, and $k_{n}, n \geq 2$, such that $\left(k_{n}+1\right) p=$ $\left(k_{n-1}+1\right) p^{*}$, by induction we obtain

$$
\left\|u_{p}\right\|_{\left(k_{n}+1\right) p^{*}} \leq S^{\frac{1}{k_{n}+1}} c^{\frac{1}{\sqrt{k_{n}+1}}}\left\|u_{p}\right\|_{\left(k_{n-1}+1\right) p^{*}}
$$

Hence, using iteratively the above inequality, we get

$$
\left\|u_{p}\right\|_{\left(k_{n}+1\right) p^{*}} \leq S^{\sum_{i=1}^{n} \frac{1}{k_{i}+1}} c^{\sum_{i=1}^{n} \frac{1}{\sqrt{k_{i}+1}}}\left\|u_{p}\right\|_{p^{*}} .
$$

Being $k_{n}+1=\left(p^{*} / p\right)^{n}$, and $p^{*} / p>1$, it follows that for any $n \geq 1$

$$
\left\|u_{p}\right\|_{\left(k_{n}+1\right) p^{*}} \leq C\|u\|_{p^{*}}
$$

as $r_{n}=\left(k_{n}+1\right) p^{*} \rightarrow+\infty$ as $n \rightarrow+\infty$. The estimates in (3.5) imply that $u \in L^{\infty}(\Omega)$. Indeed, if by contradiction the exist $\varepsilon>0$ and $A \subset \Omega$ with positive measure such that $|u|>C\|u\|_{p^{*}}+\varepsilon=K$ in $A$, we have

$$
\liminf _{n}\|u\|_{r_{n}} \geq \liminf _{n}\left(\int_{A} K^{r_{n}}\right)^{\frac{1}{r_{n}}}=K>C\|u\|_{p^{*}},
$$

which is in contrast with (3.5).

Now the $L^{\infty}$-estimate, the hypothesis (3.3) and the properties of $H$ allow to apply standard regularity results (see [23], [33]), in order to obtain that $u \in C^{1, \alpha}(\Omega)$. As matter of fact, as observed in [9] it is possible to follow the argument in [30, pages 466-467] to get the 
continuity of $u_{p}$ up to the boundary. Finally, $u_{p}$ is strictly positive in $\Omega$ by the Harnack inequality (see [34]).

Theorem 3.2. The first eigenvalue $\lambda_{1}(\Omega)$ of (3.1) is simple, that is the relative eigenfunctions are unique up to a multiplicative constant.

Proof. We follow the idea of [4,5]. Let $v, w$ two positive minimizers of (1.1) in $\Omega$ such that $\|v\|_{p}=\|w\|_{p}=1$, and consider $\eta_{t}=\left(t v^{p}+(1-t) w^{p}\right)^{1 / p}$, with $t \in[0,1]$. Obviously, $\left\|\eta_{t}\right\|_{p}=1$. Moreover, using the homogeneity and the convexity of $H$ we get that

$$
\begin{aligned}
{\left[H\left(D \eta_{t}\right)\right]^{p} } & =\eta_{t}^{p}\left[H\left(t\left(\frac{v}{\eta_{t}}\right)^{p} \frac{D v}{v}+(1-t)\left(\frac{w}{\eta_{t}}\right)^{p} \frac{D w}{w}\right)\right]^{p} \\
& =\eta_{t}^{p}\left[H\left(s(x) \frac{D v}{v}+(1-s(x)) \frac{D w}{w}\right)\right]^{p} \\
& \leq \eta_{t}^{p}\left[s(x) H\left(\frac{D v}{v}\right)+(1-s(x)) H\left(\frac{D w}{w}\right)\right]^{p} \\
& \leq t v^{p}\left[H\left(\frac{D v}{v}\right)\right]^{p}+(1-t) w^{p}\left[H\left(\frac{D w}{w}\right)\right]^{p} \\
& =t[H(D v)]^{p}+(1-t)[H(D w)]^{p} .
\end{aligned}
$$

Hence, recalling (1.2), the inequalities in (3.6) and the definition of $\eta_{t}$ give that

$$
J\left(\eta_{t}\right) \leq t J(v)+(1-t) J(w)=\lambda_{1}(\Omega),
$$

and then $\eta_{t}$ is a minimizer for $J$. This implies that the inequalities in (3.6) become equalities. The equality between the third and the fourth row of (3.6) holds if and only if $H(D v / v)=$ $H(D w / w)$. Hence, the strict convexity of the level sets of $H$ guarantees from the equalities in (3.6) that $D v / v=D w / w$ in $\Omega$, that is $v / w$ is constant. The norm constraint on $v$ and $w$ implies the uniqueness, and this concludes the proof.

Remark 3.1. We stress that the nonnegative solution $u_{p} \in C^{1, \alpha}(\Omega) \cap C(\bar{\Omega})$ of (3.1) we found by Theorem 3.1 cannot be identically zero on $\partial \Omega$. Indeed, in such a case, taking $\psi=1$ as test function in (3.2), we obtain

$$
\int_{\Omega} u_{p}^{p-1} d x=0
$$

contradicting the positivity of $u_{p}$ in $\Omega$. As a matter of fact, if we suppose $\partial \Omega$ to be a connected $C^{2}$ manifold, then the Hopf boundary point Lemma holds (see [14]), which implies that $u$ cannot vanish on $\partial \Omega$.

Theorem 3.3. Any nonnegative function $v \in W^{1, p}(\Omega), v \not \equiv 0$, which satisfies, in the sense of (3.2),

$$
\begin{cases}-\operatorname{div}\left(F_{p}(D v)\right)=\lambda v^{p-1} & \text { in } \Omega, \\ F_{p}(D v) \cdot \nu+\beta H(\nu) v^{p-1}=0 & \text { on } \partial \Omega .\end{cases}
$$

is a first eigenfunction of (3.7), that is $\lambda=\lambda_{1}(\Omega)$ and $v=u_{p}$, where $u_{p}$ is given in Theorem 3.1. up to multiplicative constant.

For analogous results in the Dirichlet case, see for example [28] and the references therein.

Proof of Theorem 3.3. The same arguments of Theorem 3.1 allow to prove that the given nonnegative solution $v$ of (3.7) is in $C^{1, \alpha}(\Omega) \cap C(\bar{\Omega})$ and it is positive in $\Omega$. Moreover, the function $u_{p} \in C^{1, \alpha}(\Omega) \cap C(\bar{\Omega})$ satisfies

$$
\int_{\Omega}\left[H\left(D u_{p}\right)\right]^{p} d x+\beta \int_{\partial \Omega} u_{p}^{p} H(\nu) d \sigma=\lambda_{1}(\Omega) \int_{\Omega} u_{p}^{p} d x
$$


while, choosing $u_{p}^{p} /(v+\varepsilon)^{p-1}$, with $\varepsilon>0$, as test function for $v$, we get

$$
\begin{aligned}
\int_{\Omega} p\left[H\left(\frac{u_{p}}{v+\varepsilon} D v\right)\right]^{p-1} H_{\xi}(D v) \cdot D u_{p} d x-(p-1) \int_{\Omega}\left[H\left(\frac{u_{p}}{v+\varepsilon} D v\right)\right]^{p} d x+ \\
+\beta \int_{\partial \Omega} \frac{v^{p-1}}{(v+\varepsilon)^{p-1}} u_{p}^{p} H(\nu) d \sigma=\lambda \int_{\Omega} \frac{v^{p-1}}{(v+\varepsilon)^{p-1}} u_{p}^{p} d x .
\end{aligned}
$$

Subtracting (3.9) by (3.8), being $H_{\xi}$ zero homogeneous, and observing that $v /(v+\varepsilon) \leq 1$, we get

$$
\begin{aligned}
\int_{\Omega}\left\{\left[H\left(D u_{p}\right)\right]^{p}-F_{p}\left(\frac{u_{p}}{v+\varepsilon} D v\right) \cdot D u_{p}+(p-1)[H\right. & \left.\left.\left(\frac{u_{p}}{v+\varepsilon} D v\right)\right]^{p}\right\} d x \leq \\
& \leq \int_{\Omega}\left[\lambda_{1}(\Omega)-\frac{v^{p-1}}{(v+\varepsilon)^{p-1}} \lambda\right] u_{p}^{p} d x .
\end{aligned}
$$

The convexity of $H^{p}$ guarantees that the left-hand side in the above inequality is nonnegative. Hence, as $\varepsilon \rightarrow 0$, the monotone convergence gives that

$$
\left(\lambda_{1}(\Omega)-\lambda\right) \int_{\Omega} u_{p}^{p} d x \geq 0
$$

and this can hold if and only if $\lambda \leq \lambda_{1}(\Omega)$. Being $\lambda_{1}(\Omega)$ the smallest possible eigenvalue, necessarily we have that $\lambda=\lambda_{1}(\Omega)$. The uniqueness of the first eigenfuction implies that, up to some positive multiplicative constant, $v=u_{p}$.

In order to show a lower bound for $\lambda_{1}(\Omega)$ when $\Omega$ is a convex set of $\mathbb{R}^{n}$ in terms of the anisotropic inradius of $\Omega$, we need an Hardy-type inequality for functions which, in general, do not vanish on the boundary. To this aim, we impose further regularity on $H$. More precisely, we assume also that

$$
\partial W=\left\{x: H^{o}(x)=1\right\} \text { has positive Gaussian curvature in any point. }
$$

If $\Omega$ is $C^{2}$, this assumption ensures that the anisotropic distance from the boundary of $\Omega$ is $C^{2}$ in a tubular neighborhood of $\partial \Omega$ (see for instance [13]).

Lemma 3.1. Let $\Omega$ be a bounded convex open set of $\mathbb{R}^{n}$ with $C^{2}$ boundary and suppose that $H^{\circ}$ satisfies also (3.10). Then, for any $\alpha>0$ and $\vartheta>0$, the following Hardy-type inequality holds:

$$
\int_{\Omega}[H(D u)]^{p} d x+\vartheta^{p-1} \int_{\partial \Omega}|u|^{p} H(\nu) d \sigma \geq(p-1)(\alpha \vartheta)^{p-1}(1-\alpha \vartheta) \int_{\Omega} \frac{|u|^{p}}{\left(d_{H}+\alpha\right)^{p}} d x,
$$

where $u \in W^{1, p}(\Omega)$ and $d_{H}$ is the anisotropic distance from the boundary of $\Omega$, defined in (2.9).

Proof. It sufficient to prove the thesis for $u \geq 0$. Moreover, using an approximation argument, we can suppose that $u \in C^{1}(\bar{\Omega})$. For $\delta$ positive, let us define $H_{\delta}(\xi)=H^{\delta}(\xi)+\delta$, where $H^{\delta}$ is the $\delta$-mollification of $H$. By the convexity of $H(\xi)$, the function $H^{\delta}$ is convex and we have, for any $\xi_{1}, \xi_{2} \in \mathbb{R}^{n}$,

$$
\left[H_{\delta}\left(\xi_{1}\right)\right]^{p} \geq\left[H_{\delta}\left(\xi_{2}\right)\right]^{p}+p\left[H_{\delta}\left(\xi_{2}\right)\right]^{p-1}\left(H_{\delta}\right)_{\xi}\left(\xi_{2}\right) \cdot\left(\xi_{1}-\xi_{2}\right) .
$$

We apply the above inequality to $\xi_{1}=D u$ and $\xi_{2}=\frac{\alpha \vartheta u}{d^{\varepsilon}+\alpha} D d^{\epsilon}$, where $\alpha>0, \vartheta>0$, and $d^{\epsilon}$ is the $\epsilon$-mollification of $d_{H}$. The convexity of $\Omega$ gives that the function $d_{H}$, and then $d^{\epsilon}$, are 
concave functions. We have:

$$
\begin{aligned}
& \int_{\Omega}\left[H_{\delta}(D u)\right]^{p} d x \geq(\alpha \vartheta)^{p} \int_{\Omega} \frac{u^{p}}{\left(d^{\epsilon}+\alpha\right)^{p}}\left[H_{\delta}\left(D d^{\epsilon}\right)\right]^{p} d x+ \\
& +p(\alpha \vartheta)^{p-1} \int_{\Omega} \frac{u^{p-1}}{\left(d^{\epsilon}+\alpha\right)^{p-1}}\left[H_{\delta}\left(D d^{\epsilon}\right)\right]^{p-1}\left(H_{\delta}\right)_{\xi}\left(D d^{\epsilon}\right) \cdot D u d x+ \\
& \quad-p(\alpha \vartheta)^{p} \int_{\Omega} \frac{u^{p}}{\left(d^{\epsilon}+\alpha\right)^{p}}\left[H_{\delta}\left(D d^{\epsilon}\right)\right]^{p-1}\left(H_{\delta}\right)_{\xi}\left(D d^{\epsilon}\right) \cdot D d^{\epsilon} d x
\end{aligned}
$$

Passing to the limit as $\delta \rightarrow 0$ and using (2.5), the sum of the first and the third terms in the right-hand side of (3.12) converge to

$$
-(p-1)(\alpha \vartheta)^{p} \int_{\Omega} \frac{u^{p}}{\left(d^{\epsilon}+\alpha\right)^{p}}\left[H\left(D d^{\epsilon}\right)\right]^{p} d x .
$$

Moreover, by the divergence theorem we have that

$$
\begin{gathered}
p \int_{\Omega} \frac{u^{p-1}}{\left(d^{\epsilon}+\alpha\right)^{p-1}}\left[H_{\delta}\left(D d^{\epsilon}\right)\right]^{p-1}\left(H_{\delta}\right)_{\xi}\left(D d^{\epsilon}\right) \cdot D u d x= \\
=\frac{1}{p} \int_{\Omega} \frac{1}{\left(d^{\epsilon}+\alpha\right)^{p-1}}\left(H_{\delta}^{p}\right)_{\xi}\left(D d^{\epsilon}\right) \cdot D\left(u^{p}\right) d x= \\
=\frac{1}{p} \int_{\partial \Omega} \frac{u^{p}}{\left(d^{\epsilon}+\alpha\right)^{p-1}}\left(H_{\delta}^{p}\right)_{\xi}\left(D d^{\epsilon}\right) \cdot \nu d \sigma-\frac{1}{p} \int_{\Omega} u^{p} \operatorname{div}\left(\frac{\left(H_{\delta}^{p}\right)_{\xi}\left(D d^{\epsilon}\right)}{\left(d^{\epsilon}+\alpha\right)^{p-1}}\right) d x= \\
=\frac{1}{p} \int_{\partial \Omega} \frac{u^{p}}{\left(d^{\epsilon}+\alpha\right)^{p-1}}\left(H_{\delta}^{p}\right)_{\xi}\left(D d^{\epsilon}\right) \cdot \nu d \sigma-\frac{1}{p} \int_{\Omega} \frac{u^{p}}{\left(d_{H}+\alpha\right)^{p-1}} \operatorname{div}\left(\left(H_{\delta}^{p}\right)_{\xi}\left(D d^{\epsilon}\right)\right) d x+ \\
\quad+\frac{p-1}{p} \int_{\Omega} \frac{u^{p}}{\left(d^{\epsilon}+\alpha\right)^{p}}\left(H_{\delta}^{p}\right)_{\xi}\left(D d^{\epsilon}\right) \cdot D d^{\epsilon} d x \geq \\
\geq \frac{1}{p} \int_{\partial \Omega} \frac{u^{p}}{\left(d^{\epsilon}+\alpha\right)^{p-1}}\left(H_{\delta}^{p}\right)_{\xi}\left(D d^{\epsilon}\right) \cdot \nu d \sigma+\frac{p-1}{p} \int_{\Omega} \frac{u^{p}}{\left(d^{\epsilon}+\alpha\right)^{p}}\left(H_{\delta}^{p}\right)_{\xi}\left(D d^{\epsilon}\right) \cdot D d^{\epsilon} d x
\end{gathered}
$$

Last inequality follows from the fact that $-\operatorname{div}\left(\left(H_{\delta}^{p}\right)_{\xi}\left(D d^{\epsilon}\right)\right)$ is nonnegative. Indeed, it is the trace of the product of the matrices $\left[\left(H_{\delta}^{p}\right)_{\xi \xi}\left(D d^{\epsilon}\right)\right]$ and $\left[-D^{2} d^{\epsilon}\right]$, which are both positive semidefinite, being $H_{\delta}^{p}$ convex and $d^{\epsilon}$ concave.

Passing to the limit as $\delta \rightarrow 0$ in (3.13), and using (2.5), we get

$$
\begin{aligned}
& p \int_{\Omega} \frac{u^{p-1}}{\left(d^{\epsilon}+\alpha\right)^{p-1}}\left[H\left(D d^{\epsilon}\right)\right]^{p-1}(H)_{\xi}\left(D d^{\epsilon}\right) \cdot D u d x \geq \\
& \quad \geq \frac{1}{p} \int_{\partial \Omega} \frac{u^{p}}{\left(d^{\epsilon}+\alpha\right)^{p-1}}\left(H^{p}\right)_{\xi}\left(D d^{\epsilon}\right) \cdot \nu d \sigma+(p-1) \int_{\Omega} \frac{u^{p}}{\left(d^{\epsilon}+\alpha\right)^{p}}\left[H\left(D d^{\epsilon}\right)\right]^{p} d x
\end{aligned}
$$

Then, as $\delta \rightarrow 0$ in (3.12), the above computations gives that

$$
\begin{aligned}
\int_{\Omega}[H(D u)]^{p} d x-\frac{(\alpha \vartheta)^{p-1}}{p} \int_{\partial \Omega} & u^{p}\left(H^{p}\right)_{\xi}\left(\frac{D d^{\epsilon}}{d^{\epsilon}+\alpha}\right) \cdot \nu d \sigma \geq \\
& \geq(p-1)(\alpha \vartheta)^{p-1}(1-\alpha \vartheta) \int_{\Omega} \frac{u^{p}}{\left(d^{\epsilon}+\alpha\right)^{p}}\left[H\left(D d^{\epsilon}\right)\right]^{p} d x .
\end{aligned}
$$

Now we pass to the limit for $\epsilon \rightarrow 0$. Recalling that under our assumptions $d_{H}$ is $C^{2}$ in a tubular neighborhood of $\partial \Omega$, by uniform convergence we get

$$
\begin{aligned}
\int_{\Omega}[H(D u)]^{p} d x-(\alpha \vartheta)^{p-1} & \int_{\partial \Omega} \frac{u^{p}}{\left(d_{H}+\alpha\right)^{p-1}}\left[H\left(D d_{H}\right)\right]^{p-1} H_{\xi}\left(D d_{H}\right) \cdot \nu d \sigma \geq \\
& \geq(p-1)(\alpha \vartheta)^{p-1}(1-\alpha \vartheta) \int_{\Omega} \frac{u^{p}}{\left(d_{H}+\alpha\right)^{p}}\left[H\left(D d_{H}\right)\right]^{p} d x .
\end{aligned}
$$


Being $H\left(D d_{H}\right)=1$ a.e. in $\Omega$, and $d_{H}=0$ on $\partial \Omega$, choosing $\vartheta^{p-1}=\beta$, and recalling that $\nu=-D d_{H} /\left|D d_{H}\right|$ on $\partial \Omega$, by (2.1) and (2.5) we get the thesis.

An immediate application of the previous Lemma is the following result.

Proposition 3.1. If $\Omega$ is a convex set of $\mathbb{R}^{n}$ with $C^{2}$ boundary and if $H$ satisfies also (3.10), then

$$
\lambda_{1}(\Omega) \geq\left(\frac{p-1}{p}\right)^{p} \frac{\beta}{R_{H, \Omega}\left(1+\beta^{\frac{1}{p-1}} R_{H, \Omega}\right)^{p-1}},
$$

where $R_{H, \Omega}$ is the anisotropic inradius of $\Omega$, as defined in (2.11).

Proof. Let $\beta=\vartheta^{p-1}$. Then, by (3.11) and the definitions of $\lambda_{1}(\Omega)$ and of the anisotropic inradius $R_{H, \Omega}$ we get that

$$
\lambda_{1}(\Omega) \geq \frac{(p-1) \beta}{\left(R_{H, \Omega}+\alpha\right)^{p}}\left(1-\beta^{\frac{1}{p-1}} \alpha\right) \alpha^{p-1} .
$$

Then, maximizing the right-hand side of the above inequality we obtain that

$$
\lambda_{1}(\Omega) \geq\left(\frac{p-1}{p}\right)^{p} \frac{\beta}{R_{H, \Omega}\left(1+\beta^{\frac{1}{p-1}} R_{H, \Omega}\right)^{p-1}} .
$$

Remark 3.2. As a consequence of the previous Proposition, we have that

$$
\sup _{|\Omega|=m} \lambda_{1}(\Omega)=+\infty \text {. }
$$

among all the Lipschitz domains with given measure $m>0$.

Finally, we have the following scaling property.

Proposition 3.2. For any $t>0$, we have that $\lambda_{1}(t \Omega, \beta)=t^{-p} \lambda_{1}\left(\Omega, t^{p-1} \beta\right)$.

Proof. By the homogeneity of $H$, we have:

$$
\begin{aligned}
\lambda_{1}(t \Omega, \beta) & =\min _{\substack{v \in W^{1, p}(t \Omega) \\
v \neq 0}} \frac{\int_{t \Omega}[H(D v(x))]^{p} d x+\beta \int_{\partial(t \Omega)}|v(x)|^{p} H(\nu(x)) d \sigma(x)}{\int_{t \Omega}|v(x)|^{p} d x} \\
& =\min _{\substack{u \in W^{1, p}(\Omega) \\
u \neq 0}} \frac{t^{n-p} \int_{\Omega}[H(D u(y))]^{p} d y+t^{n-1} \beta \int_{\partial \Omega}|u(y)|^{p} H(\nu(y)) d \sigma(y)}{t^{n} \int_{\Omega}|u(y)|^{p} d y}=t^{-p} \lambda_{1}\left(\Omega, t^{p-1} \beta\right) .
\end{aligned}
$$

\section{The eigenvalue Problem in the anisotropic Radial CaSe}

In this section we study the properties of the minimizers of (1.1) when $\Omega$ is homothetic to the Wulff shape, that is, for $R>0$, the functions $v_{p}$ such that

$$
J\left(v_{p}\right)=\min _{\substack{u \in W^{1, p}\left(\mathcal{W}_{R}\right) \\ u \neq 0}} \frac{\int_{\mathcal{W}_{R}}[H(D u)]^{p} d x+\beta \int_{\partial \mathcal{W}_{R}}|u|^{p} H(\nu) d \sigma}{\int_{\mathcal{W}_{R}}|u|^{p} d x},
$$


where $\mathcal{W}_{R}=R \mathcal{W}=\left\{x: H^{o}(x)<R\right\}$, with $R>0$, and $\mathcal{W}$ is the Wulff shape centered at the origin. By Theorem 3.1, such functions solve the following problem:

$$
\begin{cases}-\operatorname{div}\left(F_{p}(D v)\right)=\lambda_{1}\left(\mathcal{W}_{R}\right)|v|^{p-2} v & \text { in } \mathcal{W}_{R}, \\ F_{p}(D v) \cdot \nu+\beta H(\nu)|v|^{p-2} v=0 & \text { on } \partial \mathcal{W}_{R} .\end{cases}
$$

Theorem 4.1. Let $v_{p} \in C^{1, \alpha}(\Omega) \cap C(\bar{\Omega})$ be a positive solution of problem (4.2). Then, there exists a decreasing function $\varrho_{p}=\varrho_{p}(r), r \in[0, R]$, such that $\varrho_{p} \in C^{\infty}(0, R) \cap C^{1}([0, R])$, and

$$
\left\{\begin{array}{l}
v_{p}(x)=\varrho_{p}\left(H^{o}(x)\right), x \in \overline{\mathcal{W}}_{R}, \\
\varrho_{p}^{\prime}(0)=0, \\
-\left(-\varrho_{p}^{\prime}(R)\right)^{p-1}+\beta\left(\varrho_{p}(R)\right)^{p-1}=0 .
\end{array}\right.
$$

Proof. Let $B_{R}$ be the Euclidean ball centered at the origin, $B_{R}=\left\{x \in \mathbb{R}^{n}:|x|<R\right\}$, and consider the $p$-Laplace eigenvalue problem in $B_{R}$, that is (4.2) with $H(\xi)=|\xi|$ :

$$
\begin{cases}-\Delta_{p} w=\lambda_{1, \mathcal{E}}\left(B_{R}\right) \mid w^{p-2} w & \text { in } B_{R}, \\ |D w|^{p-2} \frac{\partial w}{\partial \nu}+\beta|w|^{p-2} w=0 & \text { on } \partial B_{R},\end{cases}
$$

where $\lambda_{1, \mathcal{E}}\left(B_{R}\right)$ denotes the first eigenvalue. It is known (see, for example, [16]) that problem (4.3) admits a positive radially decreasing solution $w_{p}(x)=\varrho_{p}(|x|), 0 \leq|x| \leq R$, such that $\varrho_{p} \in C^{\infty}(0, R) \cap C^{1}([0, R])$ and verifies

$$
\left\{\begin{array}{l}
\left.-(p-1)\left(-\varrho_{p}^{\prime}(r)\right)^{p-2} \varrho_{p}^{\prime \prime}(r)+\frac{n-1}{r}\left(-\varrho_{p}^{\prime}(r)\right)^{p-1}=\lambda_{1, \mathcal{E}}\left(B_{R}\right) \varrho_{p}(r)^{p-1}, \quad r \in\right] 0, R[, \\
\varrho_{p}^{\prime}(0)=0, \\
-\left(-\varrho_{p}^{\prime}(R)\right)^{p-1}+\beta \varrho_{p}(R)^{p-1}=0 .
\end{array}\right.
$$

Let $v_{p}(x)=\varrho_{p}\left(H^{o}(x)\right), x \in \mathcal{W}_{R}$. Using properties (2.5)-(2.7), for $x \in \mathcal{W}_{R} \backslash\{0\}$ we have that

$$
H\left(D v_{p}(x)\right)=-\varrho_{p}^{\prime}\left(H^{o}(x)\right) H\left(D H^{o}(x)\right)=-\varrho_{p}^{\prime}\left(H^{o}(x)\right),
$$

and

which imply that

$$
D H\left(D v_{p}(x)\right)=-D H\left(D H^{o}(x)\right)=-\frac{x}{H^{o}(x)},
$$

$$
F_{p}\left(D v_{p}\right)=-\left(-\varrho^{\prime}\left(H^{o}(x)\right)\right)^{p-1} \frac{x}{H^{o}(x)}
$$

and then, by (4.4),

$$
\begin{aligned}
-\operatorname{div}\left(F_{p}\left(D v_{p}\right)\right) & =-(p-1)\left(-\varrho_{p}^{\prime}\left(H^{o}(x)\right)\right)^{p-2} \varrho_{p}^{\prime \prime}\left(H^{o}(x)\right)+\frac{n-1}{H^{o}(x)}\left(-\varrho_{p}^{\prime}\left(H^{o}(x)\right)\right)^{p-1} \\
& =\lambda_{1, \mathcal{E}}\left(B_{R}\right) v_{p}(x)^{p-1} \quad \text { for } x \in \mathcal{W}_{R} \backslash\{0\} .
\end{aligned}
$$

As regards the boundary condition, observing that $\nu(x)=D H^{o}(x) /\left|D H^{\circ}(x)\right|$, by (4.5), the properties (2.5), (2.6), and (4.4) we have that

$$
\begin{aligned}
F_{p}\left(v_{p}(x)\right) \cdot \nu(x)+\beta H(\nu(x)) v_{p}(x)^{p-1} & =\frac{1}{\left|D H^{o}(x)\right|}\left(-\left(-\varrho^{\prime}(R)\right)^{p-1}+\beta \varrho_{p}(R)^{p-1}\right) \\
& =0 \quad \text { for } x \in \partial \mathcal{W}_{R} .
\end{aligned}
$$

Hence, integrating (4.6) on $\mathcal{W}_{R} \backslash \mathcal{W}_{\varepsilon}$, we can use the divergence theorem and the boundary condition (4.7), and let $\varepsilon$ going to 0 , obtaining that $v_{p}$ verifies

$$
\begin{cases}-\operatorname{div}\left(F_{p}\left(D v_{p}\right)\right)=\lambda_{1, \mathcal{E}}\left(B_{R}\right) v_{p}^{p-1} & \text { in } \mathcal{W}_{R}, \\ F_{p}(D v) \cdot \nu+\beta H(\nu) v_{p}^{p-1}=0 & \text { on } \partial \mathcal{W}_{R} .\end{cases}
$$


But Theorem 3.3 guarantees that a positive solution of (4.8) has to be a first eigenfunction, and

$$
\lambda_{1}\left(\mathcal{W}_{R}\right)=\lambda_{1, \mathcal{E}}\left(B_{R}\right)
$$

This concludes the proof.

Remark 4.1. We observe that the proof of the above theorem shows that, for any convex function $H$ we can consider, the first eigenvalue in the ball $\mathcal{W}_{R}=\left\{H^{o}(x)<R\right\}$ is the same, and coincides with the first eigenvalue for the $p$-Laplacian problem (4.3) in the Euclidean ball $B_{R}$ (with the same $R$ ).

Next two lemmata will be useful in the proof of the main result. Their proofs are analogous to the ones obtained in [9]. For the sake of completeness, we write them in details.

Lemma 4.1. If $0<r<s$, then $\lambda_{1}\left(\mathcal{W}_{r}\right)>\lambda_{1}\left(\mathcal{W}_{s}\right)$.

Proof. Let $v_{p}$ a minimizer of (4.1), with $R=r$, and take $w(x)=v_{p}\left(\frac{r}{s} x\right), x \in \mathcal{W}_{s}$. Then, by the homogeneity of $H$ we get

$$
\begin{aligned}
\lambda_{1}\left(\mathcal{W}_{s}\right) & \leq \frac{\int_{\mathcal{W}_{s}}[H(D w)]^{p} d x+\beta \int_{\partial \mathcal{W}_{s}}|w|^{p} H(\nu) d \sigma}{\int_{\mathcal{W}_{s}}|w|^{p} d x} \\
& =\frac{\left(\frac{r}{s}\right)^{p} \int_{\mathcal{W}_{r}}\left[H\left(D v_{p}\right)\right]^{p} d x+\beta \frac{r}{s} \int_{\partial \mathcal{W}_{r}}\left|v_{p}\right|^{p} H(\nu) d \sigma}{\int_{\mathcal{W}_{r}}\left|v_{p}\right|^{p} d x} \\
& <\frac{\int_{\mathcal{W}_{r}}\left[H\left(D v_{p}\right)\right]^{p} d x+\beta \int_{\partial \mathcal{W}_{r}}\left|v_{p}\right|^{p} H(\nu) d \sigma}{\int_{\mathcal{W}_{r}}\left|v_{p}\right|^{p} d x}=\lambda_{1}\left(W_{r}\right)
\end{aligned}
$$

We stress that by (4.4), if $v_{p}(x)=\varrho_{p}\left(H^{o}(x)\right)$ is the positive solution in $\mathcal{W}_{R}$ we found in Theorem 4.1, we have that, for $x \in \partial \mathcal{W}_{R}$,

$$
\beta=\frac{\left[H\left(D v_{p}(x)\right)\right]^{p-1}}{v_{p}(x)^{p-1}} .
$$

Then, for every $0 \leq r \leq R$, we define

$$
\beta_{r}=\frac{\left[H\left(D v_{p}(x)\right)\right]^{p-1}}{v_{p}(x)^{p-1}}, \quad \text { for } H^{o}(x)=r .
$$

Let us observe that $\beta_{0}=0$ and $\beta_{R}=\beta$.

Lemma 4.2. If $0 \leq r<s \leq R$, then $\beta_{r}<\beta_{s}$.

Proof. We first observe that, similarly as in the proof of Theorem 4.1, for $0<r<R$, the function $v_{p}$ is such that

$$
\begin{cases}-\operatorname{div}\left(F_{p}\left(D v_{p}\right)\right)=\lambda_{1}\left(\mathcal{W}_{R}\right) v_{p}^{p-1} & \text { in } \mathcal{W}_{r}, \\ F_{p}\left(D v_{p}\right) \cdot \nu+\beta_{r} H(\nu) v_{p}^{p-1}=0 & \text { on } \partial \mathcal{W}_{r}\end{cases}
$$


Then, denoted by $\lambda_{1}\left(\mathcal{W}_{r}, \beta_{r}\right)$ the first eigenvalue in $\mathcal{W}_{r}$ with $\beta=\beta_{r}$, by Theorem 3.3 we have necessarily $\lambda_{1}\left(\mathcal{W}_{R}\right)=\lambda_{1}\left(\mathcal{W}_{r}, \beta_{r}\right)$ for all $\left.\left.r \in\right] 0, R\right]$. Hence, by Lemma 4.1 we obtain, for $0<r<s \leq R$, that

$$
\begin{aligned}
\frac{\int_{\mathcal{W}_{r}}\left[H\left(D v_{p}\right)\right]^{p} d x+\beta_{r} \int_{\partial \mathcal{W}_{r}} v_{p}^{p} H(\nu) d \sigma}{\int_{\mathcal{W}_{r}} v_{p}^{p} d x}=\lambda_{1}\left(\mathcal{W}_{r}, \beta_{r}\right)=\lambda_{1}\left(\mathcal{W}_{s}, \beta_{s}\right)<\lambda_{1}\left(\mathcal{W}_{r}, \beta_{s}\right) \leq \\
\leq \frac{\int_{\mathcal{W}_{r}}\left[H\left(D v_{p}\right)\right]^{p} d x+\beta_{s} \int_{\partial \mathcal{W}_{r}} v_{p}^{p} H(\nu) d \sigma}{\int_{\mathcal{W}_{r}} v_{p}^{p} d x},
\end{aligned}
$$

and then $\beta_{r}<\beta_{s}$.

\section{A REpresentation Formula FOR $\lambda_{1}(\Omega)$}

Now we prove a level set representation formula for the first eigenvalue $\lambda_{1}(\Omega)$. To this aim, we will use the following notation. Let $\tilde{u}_{p}$ be the first positive eigenfunction such that $\max \tilde{u}_{p}=1$. Then, for $t \in[0,1]$,

$$
\begin{aligned}
& U_{t}=\left\{x \in \Omega: \tilde{u}_{p}>t\right\}, \\
& S_{t}=\left\{x \in \Omega: \tilde{u}_{p}=t\right\}, \\
& \Gamma_{t}=\left\{x \in \partial \Omega: \tilde{u}_{p}>t\right\} .
\end{aligned}
$$

First of all, it is worth to observe that the anisotropic areas of the sets $\partial U_{t}, S_{t}$ and $\Gamma_{t}$, defined in 2.1. are related in the following way.

Lemma 5.1. There exists a countable set $\mathcal{Q} \subset] 0,1[$ such that

$$
\left.\sigma_{H}\left(\partial U_{t}\right) \leq \sigma_{H}\left(\Gamma_{t}\right)+\sigma_{H}\left(S_{t}\right), \quad \forall t \in\right] 0,1[\backslash \mathcal{Q} .
$$

Proof. The proof follows similarly as in [9]. The continuity up to the boundary of the eigenfunction $\tilde{u}_{p}$, given in Theorem 3.1, guarantees that

$$
\partial U_{t} \cap \Omega \subseteq S_{t}, \quad \partial U_{t} \cap \partial \Omega \subseteq \tilde{\Gamma}_{t}
$$

for any $t \in[0,1]$, where $\tilde{\Gamma}_{t}=\left\{x \in \partial \Omega: \tilde{u}_{p} \geq t\right\}$. Moreover, by [32, Section 1.2.3] we have that

$$
\int_{0}^{\infty} \sigma_{H}\left(\Gamma_{t}\right) d t=\int_{0}^{\infty} \sigma_{H}\left(\tilde{\Gamma}_{t}\right) d t=\int_{\partial \Omega} \tilde{u} d \sigma_{H} \leq \sigma_{H}(\partial \Omega)<+\infty
$$

Hence $\sigma_{H}\left(\Gamma_{t}\right) \leq \sigma_{H}\left(\tilde{\Gamma}_{t}\right)<+\infty$ and then $\sigma_{H}\left(\Gamma_{t}\right)=\sigma_{H}\left(\tilde{\Gamma}_{t}\right)$ for a.e. $t \in[0,1]$. Moreover, being $\sigma_{H}\left(\Gamma_{t}\right)$ and $\sigma_{H}\left(\tilde{\Gamma}_{t}\right)$ monotone decreasing in $t$, they are continuous in $[0,1]$ up to a countable set $\mathcal{Q}$. Hence,

$$
\sigma_{H}\left(\partial U_{t}\right)=\sigma_{H}\left(\partial U_{t} \cap \Omega\right)+\sigma_{H}\left(\partial U_{t} \cap \partial \Omega\right) \leq \sigma_{H}\left(S_{t}\right)+\sigma_{H}\left(\Gamma_{t}\right)
$$

for all $t \in[0,1] \backslash \mathcal{Q}$. 
If we formally divide both terms in the equation in (3.1) by $\tilde{u}_{p}^{p-1}$, and integrate in $U_{t}$, by (2.5) and the boundary condition we get

$$
\begin{aligned}
& \lambda_{1}(\Omega)\left|U_{t}\right|=\int_{U_{t}} \frac{-\operatorname{div}\left(F_{p}\left(D \tilde{u}_{p}\right)\right)}{\tilde{u}_{p}^{p-1}} d x= \\
& =-(p-1) \int_{U_{t}} \frac{\left[H\left(D \tilde{u}_{p}\right)\right]^{p-1} H_{\xi}\left(D \tilde{u}_{p}\right) \cdot D \tilde{u}_{p}}{\tilde{u}_{p}^{p}} d x-\int_{\partial U_{t}} \frac{\left[H\left(D \tilde{u}_{p}\right)\right]^{p-1}}{\tilde{u}_{p}^{p-1}} H_{\xi}\left(D \tilde{u}_{p}\right) \cdot \nu d \sigma= \\
& =-(p-1) \int_{U_{t}} \frac{\left[H\left(D \tilde{u}_{p}\right)\right]^{p}}{\tilde{u}_{p}^{p}} d x+\int_{S_{t}} \frac{\left[H\left(D \tilde{u}_{p}\right)\right]^{p-1}}{\tilde{u}_{p}^{p-1}} H(\nu) d \sigma+\beta \int_{\Gamma_{t}} H(\nu) d \sigma= \\
& =\left|U_{t}\right| \mathcal{F}_{\Omega}\left(U_{t}, \frac{\left[H\left(D \tilde{u}_{p}\right)\right]^{p-1}}{\tilde{u}_{p}^{p-1}}\right),
\end{aligned}
$$

where

$$
\mathcal{F}_{\Omega}\left(U_{t}, \varphi\right)=\frac{1}{\left|U_{t}\right|}\left(-(p-1) \int_{U_{t}} \varphi^{p^{\prime}} d x+\int_{S_{t}} \varphi H(\nu) d \sigma+\beta \int_{\Gamma_{t}} H(\nu) d \sigma\right),
$$

with $\varphi$ nonnegative measurable function in $\Omega$. The formal computations in (5.2) give a representation formula of $\lambda_{1}(\Omega)$ which will be rigorously proved in the result below.

Theorem 5.1. Let $\tilde{u}_{p} \in C^{1, \alpha}(\Omega) \cap C(\bar{\Omega})$ be the positive minimizer of (1.1) such that $\max \tilde{u}_{p}=1$. Then, for a.e. $\left.t \in\right] 0,1[$,

$$
\lambda_{1}(\Omega)=\mathcal{F}_{\Omega}\left(U_{t}, \frac{\left[H\left(D \tilde{u}_{p}\right)\right]^{p-1}}{\tilde{u}_{p}^{p-1}}\right) .
$$

Proof. Let $0<\varepsilon<t<1$, and

$$
\psi_{\varepsilon}= \begin{cases}0 & \text { if } \tilde{u}_{p} \leq t \\ \frac{u-t}{\varepsilon} \frac{1}{\tilde{u}_{p}^{p-1}} & \text { if } t<\tilde{u}_{p}<t+\varepsilon \\ \frac{1}{\tilde{u}_{p}^{p-1}} & \text { if } \tilde{u}_{p} \geq t+\varepsilon .\end{cases}
$$

The functions $\psi_{\varepsilon}$ are in $W^{1, p}(\Omega)$ and increasingly converge to $\tilde{u}_{p}^{-(p-1)} \chi_{U_{t}}$ as $\varepsilon \searrow 0$. Moreover,

$$
D \psi_{\varepsilon}= \begin{cases}0 & \text { if } \tilde{u}_{p}<t \\ \frac{1}{\varepsilon}\left((p-1) \frac{t}{\tilde{u}_{p}}+2-p\right) \frac{D \tilde{u}_{p}}{\tilde{u}_{p}^{p-1}} & \text { if } t<u_{p}<t+\varepsilon \\ -(p-1) \frac{D \tilde{u}_{p}}{\tilde{u}_{p}^{p}} & \text { if } \tilde{u}_{p}>t+\varepsilon .\end{cases}
$$

Then, choosing $\psi_{\varepsilon}$ as test function in (3.2), we get that the first integral is

$$
\begin{aligned}
& -(p-1) \int_{U_{t+\varepsilon}} \frac{\left[H\left(D \tilde{u}_{p}\right)\right]^{p}}{\tilde{u}_{p}^{p}} d x+\frac{1}{\varepsilon} \int_{U_{t} \backslash U_{t+\varepsilon}} \frac{\left[H\left(D \tilde{u}_{p}\right)\right]^{p}}{\tilde{u}_{p}^{p-1}}\left((p-1) \frac{t}{\tilde{u}_{p}}+2-p\right) d x= \\
& =-(p-1) \int_{U_{t+\varepsilon}} \frac{\left[H\left(D \tilde{u}_{p}\right)\right]^{p}}{\tilde{u}_{p}^{p}} d x+\frac{1}{\varepsilon} \int_{t}^{t+\varepsilon}\left((p-1) \frac{t}{\tau}+2-p\right) \int_{S_{\tau}} \frac{\left[H\left(D \tilde{u}_{p}\right)\right]^{p-1}}{\tilde{u}_{p}^{p-1}} H(\nu) d \sigma,
\end{aligned}
$$

where last equality follows by the coarea formula. Then, reasoning similarly as in [9], we get that

$$
\int_{\Omega}\left[H\left(D \tilde{u}_{p}\right)\right]^{p-1} H_{\xi}\left(D \tilde{u}_{p}\right) \cdot D \psi_{\varepsilon} d x \stackrel{\varepsilon \rightarrow 0}{\longrightarrow}-(p-1) \int_{U_{t}} \frac{\left[H\left(D \tilde{u}_{p}\right)\right]^{p}}{\tilde{u}_{p}^{p}} d x+\int_{S_{t}} \frac{\left[H\left(D \tilde{u}_{p}\right)\right]^{p-1}}{\tilde{u}_{p}^{p-1}} H(\nu) d \sigma .
$$


As regards the other two integrals in (3.2), we have that

$$
\beta \int_{\partial \Omega} \tilde{u}_{p}^{p-1} \psi_{\varepsilon} H(\nu) d \sigma=\beta \int_{\Gamma_{t+\varepsilon}} H(\nu) d \sigma+\beta \int_{\Gamma_{t} \backslash \Gamma_{t+\varepsilon}} \frac{u-t}{\varepsilon} H(\nu) d \sigma \stackrel{\varepsilon \rightarrow 0}{\longrightarrow} \beta \int_{\Gamma_{t}} H(\nu) d \sigma,
$$

and, by monotone convergence theorem and the definition of $\psi_{\varepsilon}$,

$$
\lambda_{1}(\Omega) \int_{\Omega} \tilde{u}_{p}^{p-1} \psi_{\varepsilon} d x \stackrel{\varepsilon \rightarrow 0}{\longrightarrow} \lambda_{1}(\Omega)\left|U_{t}\right| .
$$

Summing the three limits, we get (5.4).

Theorem 5.2. Let $\varphi$ be a nonnegative function in $\Omega$ such that $\varphi \in L^{p^{\prime}}(\Omega)$. If $\varphi \not \equiv$ $\left[H\left(D \tilde{u}_{p}\right)\right]^{p-1} / \tilde{u}_{p}^{p-1}$, where $\tilde{u}_{p}$ is the eigenfunction given in Theorem $\left[5.1\right.$, and $\mathcal{F}_{\Omega}$ is the functional defined in (5.3), then there exists a set $S \subset] 0,1[$ with positive measure such that for every $t \in S$ it holds that

$$
\lambda_{1}(\Omega)>\mathcal{F}_{\Omega}\left(U_{t}, \varphi\right) .
$$

Proof. The proof is similar to the one obtained in [9], and we only sketch it here. It can be divided in two main steps. First, we claim that, if

$$
w(x):=\varphi-\frac{\left[H\left(D \tilde{u}_{p}\right)\right]^{p-1}}{\tilde{u}_{p}^{p-1}}, \quad I(t):=\int_{U_{t}} w \frac{H\left(D \tilde{u}_{p}\right)}{\tilde{u}_{p}} d x,
$$

then $I:] 0,1[\rightarrow \mathbb{R}$ is locally absolutely continuous and

$$
\mathcal{F}_{\Omega}\left(U_{t}, \varphi\right) \leq \lambda_{1}(\Omega)-\frac{1}{\left|U_{t}\right| t^{p-1}}\left(\frac{d}{d t} t^{p} I(t)\right),
$$

for almost every $t \in] 0,1[$. Second, we show that the derivative in (5.6) is strictly positive in a subset of ]0,1[ of positive measure.

In order to prove (5.6), writing the representation formula (5.4) in terms of $w$, it follows that, for a.e. $t \in] 0,1[$,

$$
\begin{aligned}
\mathcal{F}_{\Omega}\left(U_{t}, \varphi\right) & =\lambda_{1}(\Omega)+\frac{1}{\left|U_{t}\right|}\left(\int_{S_{t}} w H(\nu) d \sigma-(p-1) \int_{U_{t}}\left(\varphi^{p^{\prime}}-\frac{\left[H\left(D \tilde{u}_{p}\right)\right]^{p}}{\tilde{u}_{p}^{p}}\right) d x\right) \\
& \leq \lambda_{1}(\Omega)+\frac{1}{\left|U_{t}\right|}\left(\int_{S_{t}} w H(\nu) d \sigma-p \int_{U_{t}} w \frac{H\left(D \tilde{u}_{p}\right)}{\tilde{u}_{p}} d x\right) \\
& =\lambda_{1}(\Omega)+\frac{1}{\left|U_{t}\right|}\left(\int_{S_{t}} w H(\nu) d \sigma-p I(t)\right)
\end{aligned}
$$

where the inequality in (5.7) follows from the inequality $\varphi^{p^{\prime}} \geq v^{p^{\prime}}+p^{\prime} v^{p^{\prime}-1}(\varphi-v)$, with $\varphi, v \geq 0$. Applying the coarea formula, it is possible to rewrite $I(t)$ as

$$
I(t)=\int_{U_{t}} w \frac{H\left(D \tilde{u}_{p}\right)}{\tilde{u}_{p}} d x=\int_{t}^{1} \frac{1}{\tau} d \tau \int_{S_{\tau}} w H(\nu) d \sigma .
$$

This assures that $I(t)$ is locally absolutely continuous in $] 0,1[$ and, for almost every $t \in] 0,1[$ we have

$$
-\frac{d}{d t}\left(t^{p} I(t)\right)=t^{p-1}\left(\int_{S_{t}} w H(\nu) d \sigma-p I(t)\right) .
$$

Substituting in (5.7), the inequality (5.6) follows. In order to conclude the proof, arguing by contradiction exactly as in [9, Theorem 3.2], it is possible to show that $G(t):=t^{p} I(t)$ has positive derivative in a set of positive measure. Together with (5.6), this implies (5.5) . 


\section{MAIN RESUlT}

Now we are in position to state and prove the desired Faber-Krahn inequality.

Theorem 6.1. Let $\Omega \subset \mathbb{R}^{n}, n \geq 2$, be a bounded Lipschitz domain, and $H: \mathbb{R}^{n} \rightarrow[0,+\infty[$ a function with strictly convex sublevel sets which satisfies (2.1), (2.2), and (3.3). Then,

$$
\lambda_{1}(\Omega) \geq \lambda_{1}\left(\mathcal{W}_{R}\right)
$$

where $\mathcal{W}_{R}$ is the Wulff shape centered at the origin such that $\left|\mathcal{W}_{R}\right|=|\Omega|$. The equality holds if and only if $\Omega$ is a Wulff shape.

Proof. The first step in order to prove the result is to construct a suitable test function in $\Omega$ for (5.3). Let $v_{p}$ be a positive eigenfunction of the anisotropic radial problem (4.2) in $\mathcal{W}_{R}$. By Theorem 4.1, $v_{p}$ is a function depending only by $H^{o}(x)$, and then we are able to define, as in (4.9), the function

$$
\beta_{r}=\varphi_{\star}(x)=\frac{\left[H\left(D v_{p}(x)\right)\right]^{p-1}}{v_{p}(x)^{p-1}}, \quad \text { with } x \in \overline{\mathcal{W}}_{R} \text {, i.e. } H^{o}(x)=r \in[0, R] .
$$

As before, let $\tilde{u}_{p}$ be the first eigenfunction of (3.1) in $\Omega$ such that $\left\|\tilde{u}_{p}\right\|_{\infty}=1$. Using the same notation of Section 5 , for any $t \in] 0,1\left[\right.$ we consider $\mathcal{W}_{r(t)}$, the Wulff shape centered at the origin, where $r(t)$ is the positive number such that $\left|U_{t}\right|=\left|\mathcal{W}_{r(t)}\right|$. Then, for $x \in \Omega$ and $\tilde{u}_{p}(x)=t$, we define

$$
\varphi(x):=\beta_{r(t)} .
$$

Similarly as in [9], $\varphi$ is a measurable function. Thanks to this test function, we can compare $\mathcal{F}_{\Omega}\left(U_{t}, \varphi\right)$ with $\mathcal{F}_{\mathcal{W}_{R}}\left(B_{r(t)}, \varphi_{\star}\right)$. Indeed, we claim that

$$
\begin{aligned}
\mathcal{F}_{\Omega}\left(U_{t}, \varphi\right) & \geq \frac{1}{\left|\mathcal{W}_{r(t)}\right|}\left(-(p-1) \int_{\mathcal{W}_{r(t)}} \varphi_{\star}^{p^{\prime}} d x+\int_{\partial \mathcal{W}_{r}} \varphi_{\star} H(\nu) d \sigma\right) \\
& =\mathcal{F}_{\mathcal{W}_{R}}\left(\mathcal{W}_{r(t)}, \varphi_{\star}\right)
\end{aligned}
$$

for all $t \in] 0,1[\backslash \mathcal{Q}$, where $\mathcal{Q}$ is the set of Lemma 5.1. In order to show (6.2), we first observe that by [32, Section 1.2.3], being $\left|U_{t}\right|=\left|\mathcal{W}_{r(t)}\right|$ for all $\left.t \in\right] 0,1[$

$$
\int_{U_{t}} \varphi^{p^{\prime}} d x=\int_{\mathcal{W}_{r(t)}} \varphi_{\star}^{p^{\prime}} d x
$$

Moreover, the anisotropic isoperimetric inequality (2.8), Lemma 5.1 and being, by Lemma 4.2. $\beta_{r(t)} \leq \beta$ for any $t$, we have that

$$
\begin{aligned}
\int_{\partial \mathcal{W}_{r(t)}} & \varphi_{\star} H(\nu) d \sigma=\beta_{r(t)} \sigma_{H}\left(\partial \mathcal{W}_{r(t)}\right) \leq \\
& \leq \beta_{r(t)} \sigma_{H}\left(\partial U_{t}\right) \leq \beta_{r(t)} \sigma_{H}\left(S_{t}\right)+\beta_{r(t)} \sigma_{H}\left(\Gamma_{t}\right) \leq \int_{S_{t}} \varphi H(\nu) d \sigma+\beta \int_{\Gamma_{t}} H(\nu) d \sigma .
\end{aligned}
$$

Hence, joining (6.3) and (6.4) we get (6.2). Then, applying the level set representation formula (5.4) in the anisotropic radial case, and (5.5), by (6.2) we get

$$
\lambda_{1}\left(\mathcal{W}_{R}\right)=\mathcal{F}_{\mathcal{W}_{R}}\left(\mathcal{W}_{r(t)}, \varphi_{\star}\right) \leq \mathcal{F}_{\Omega}\left(U_{t}, \varphi\right) \leq \lambda_{1}(\Omega)
$$

for some $t \in] 0,1[$, which gives (6.1).

In order to conclude the proof, we study the equality case. Let us suppose that $\lambda_{1}(\Omega)=$ $\lambda_{1}\left(\mathcal{W}_{R}\right)$.

We first claim that, for a.e. $t \in] 0,1\left[, U_{t}\right.$ is homothetic to a Wulff shape. Indeed, by (5.5) and (6.2)

$$
\lambda_{1}\left(\mathcal{W}_{R}\right)=\lambda_{1}(\Omega) \geq \mathcal{F}_{\Omega}\left(U_{t}, \varphi\right) \geq \mathcal{F}_{\mathcal{W}_{R}}\left(\mathcal{W}_{r(t)}, \varphi_{\star}\right)=\lambda_{1}\left(\mathcal{W}_{R}\right)
$$


for $t$ in a set of positive measure $S \subset] 0,1[$. Then by Theorems 5.1 and 5.2 it follows necessarily that $\varphi=\frac{H\left(D \tilde{u}_{p}\right)^{p-1}}{\tilde{u}_{p}^{p-1}}$. This implies that for almost every $\left.t \in\right] 0,1[$, the equality in (6.2) and in (6.4) holds. In particular, $\sigma_{H}\left(\partial \mathcal{W}_{r(t)}\right)=\sigma_{H}\left(\partial U_{t}\right)$ for a.e. $t$. By the equality case in the anisotropic isoperimetric inequality, we get the claim. Since $\left.U_{t}, t \in\right] 0,1[$ are nested sets all homothetic to Wulff shapes, it follows that also $\Omega=\bigcup_{t \in] 0,1[} U_{t}$ is homothetic to a Wulff shape, up to a measure zero set. The Lipschitz assumption on the boundary of $\Omega$ guarantees that $\Omega=\mathcal{W}_{R}$, up to translations.

\section{REFERENCES}

[1] A. Alvino, V. Ferone, P.-L. Lions, and G. Trombetti. Convex symmetrization and applications. Ann. Inst. H. Poincaré Anal. Non Linéaire, 14(2):275-293, 1997.

[2] B. Andrews. Volume-preserving Anisotropic Mean Curvature Flow. Indiana Univ. Math. J., 50(2):783$827,2001$.

[3] G. Bellettini and M. Paolini. Anisotropic motion by mean curvature in the context of Finsler geometry. Hokkaido Math. J., 25:537-566, 1996.

[4] M. Belloni, V. Ferone, and B. Kawohl. Isoperimetric inequalities, Wulff shape and related questions for strongly nonlinear elliptic operators. Zeitschrift fur Angewandte Mathematik und Physik (ZAMP), 54(5):771-783, 2003.

[5] M. Belloni and B. Kawohl. A direct uniqueness proof for equations involving the $p$-Laplace operator. Manuscripta Math., 109(2):229-231, 2002.

[6] M. Belloni, B. Kawohl, and P. Juutinen. The $p$-Laplace eigenvalue problem as $p \rightarrow \infty$ in a Finsler metric. J. Eur. Math. Soc. (JEMS), 8(1):123-138, 2006.

[7] M.-H. Bossel. Membranes ĺastiquement liés: extension du thórème de Rayleigh-Faber-Krahn et de l'inǵalité de Cheeger. C. R. Acad. Sci. Paris Sér. I Math., 302(1):47-50, 1986.

[8] B. Brandolini, F. Chiacchio, and C. Trombetti. Optimal lower bounds for eigenvalues of linear and nonlinear Neumann problems. http://arxiv.org/abs/1302.1795, pages 1-14.

[9] D. Bucur and D. Daners. An alternative approach to the Faber-Krahn inequality for Robin problems. Calc. Var., 37:75-86, 2010.

[10] H. Busemann. The Isoperimetric Problem for Minkowski Area. Amer. J. Math., 71:743-762, 1949.

[11] F. Chiacchio and G. Di Blasio. Isoperimetric inequalities for the first Neumann eigenvalue in Gauss space. Annales de l'Institut Henri Poincaré (C) Non Linear Analysis, 29(2):199-216, Mar. 2012.

[12] A. Cianchi and P. Salani. Overdetermined anisotropic elliptic problems. Math. Ann., 345(4):859-881, 2009.

[13] G. Crasta and A. Malusa. The distance function from the boundary in a Minkowski space. Trans. Amer. Math. Soc., 359(12):5725-5759, 2007.

[14] M. Cuesta and P. Takáč. A strong comparison principle for positive solutions of degenerate elliptic equations. Differential Integral Equations, 13:721-746, 2000.

[15] B. Dacorogna and C.-E. Pfister. Wulff theorem and best constant in Sobolev inequality. J. Math. Pures Appl. (9), 71(2):97-118, 1992.

[16] Q.-y. Dai and Y.-x. Fu. Faber-Krahn inequality for Robin problems involving $p$-Laplacian. Acta Mathematica Applicatae Sinica, English Series, 27:13-28, 2011.

[17] D. Daners. A Faber-Krahn inequality for Robin problems in any space dimension. Mathematische Annalen, 335:767-785, June 2006.

[18] F. Della Pietra and N. Gavitone. Symmetrization for Neumann anisotropic problems and related questions. Advanced Nonlinear Stud., 12(2):219-235, 2012.

[19] F. Della Pietra and N. Gavitone. Anisotropic elliptic equations with general growth in the gradient and Hardy-type potentials. Journal of Differential Equations, 255:3788-3810, 2013.

[20] F. Della Pietra and N. Gavitone. Anisotropic elliptic problems involving Hardy-type potentials. J. Math. Anal. Appl., 397(2):800-813, 2013.

[21] F. Della Pietra and N. Gavitone. Relative isoperimetric inequality in the plane: the anisotropic case. $J$. Convex. Anal., 20(1):157-180, 2013.

[22] F. Della Pietra and N. Gavitone. Sharp bounds for the first eigenvalue and the torsional rigidity related to some anisotropic operators. Math. Nachr., 2013.

[23] E. DiBenedetto. $C^{1+\alpha}$ local regularity of weak solutions of degenerate elliptic equations. Nonlinear Anal., $7(8): 827-850,1983$.

[24] V. Ferone and B. Kawohl. Remarks on a Finsler-Laplacian. Proc. Am. Math. Soc., 137(1):247-253, 2009. 
[25] V. Ferone, C. Nitsch, and C. Trombetti. On a conjectured reverse Faber-Krahn inequality for a Steklovtype Laplacian eigenvalue. arXiv:1307.3788, pages 1-22, 2013.

[26] I. Fonseca and S. Müller. A uniqueness proof for the Wulff theorem. Proc. Roy. Soc. Edinburgh Sect. A, 119(1-2):125-136, 1991.

[27] D. Gilbarg and N. S. Trudinger. Elliptic partial differential equations of second order. Springer-Verlag, second edition, 1983.

[28] B. Kawohl, M. Lucia, and S. Prashanth. Simplicity of the principal eigenvalue for indefinite quasilinear problems. Adv. Differential Equations, 12(4):407-434, 2007.

[29] H. Kovařík. On the Lowest Eigenvalue of Laplace Operators with Mixed Boundary Conditions. Journal of Geometric Analysis DOI: 10.1007/s12220-012-9383-4, pages 1-17, 2012.

[30] O. A. Ladyzhenskaya and N. N. Ural'tseva. Linear and quasilinear elliptic equations. Translated from the Russian by Scripta Technica, Inc. Translation editor: Leon Ehrenpreis. Academic Press, New York, 1968.

[31] A. Lê. Eigenvalue problems for the p-Laplacian. Nonlinear Analysis: T.M.A., 64(5):1057-1099, 2006.

[32] V. G. Maz'ya. Sobolev spaces. Springer Verlag, Berlin, 1985.

[33] P. Tolksdorf. Regularity for a more general class of quasilinear elliptic equations. J. Differential Equations, 51(1):126-150, 1984.

[34] N. Trudinger. On Harnack type inequalities and their application to quasilinear elliptic equations. Comm. Pure Appl. Math., 20:721-747, 1967.

[35] G. Wang and C. Xia. A Characterization of the Wulff Shape by an Overdetermined Anisotropic PDE. Archive for Rational Mechanics and Analysis, 199(1):99-115, Apr. 2010.

[36] G. Wang and C. Xia. An optimal anisotropic Poincaré inequality for convex domains. Pacific J. Math., 258(2):305-326, 2012.

[37] H. Weinberger. An Isoperimetric Inequality for the $N$-dimensional free membrane problem. J. Rational Mech. Anal., 5(4):633-636, 1956.

Francesco Della Pietra, Università degli studi di Napoli "Federico iI", Dipartimento di Matematica e Applicazioni "R. Caccioppoli", Complesso di Monte Sant'Angelo, Via Cintia, 80126 NAPOLI, ITALIA.

E-mail address: f.dellapietra@unina.it

Nunzia Gavitone, Università degli studi di Napoli "Federico II", Dipartimento di MatematiCa e Applicazioni "R. Caccioppoli", Complesso di Monte Sant'Angelo, Via Cintia, 80126 Napoli, ITALIA.

E-mail address: nunzia.gavitone@unina.it 